Louisiana State University

LSU Digital Commons

Faculty Publications

Department of Biological Sciences

$10-1-2015$

\title{
Host promiscuity in symbiont associations can influence exotic legume establishment and colonization of novel ranges
}

\author{
Metha M. Klock \\ Louisiana State University \\ Luke G. Barrett \\ CSIRO Agriculture Flagship, Brisbane \\ Peter $\mathrm{H}$. Thrall \\ CSIRO Agriculture Flagship, Brisbane \\ Kyle E. Harms \\ Louisiana State University
}

Follow this and additional works at: https://digitalcommons.Isu.edu/biosci_pubs

\section{Recommended Citation}

Klock, M., Barrett, L., Thrall, P., \& Harms, K. (2015). Host promiscuity in symbiont associations can influence exotic legume establishment and colonization of novel ranges. Diversity and Distributions, 21 (10), 1193-1203. https://doi.org/10.1111/ddi.12363

This Article is brought to you for free and open access by the Department of Biological Sciences at LSU Digital Commons. It has been accepted for inclusion in Faculty Publications by an authorized administrator of LSU Digital Commons. For more information, please contact ir@lsu.edu. 
San Jose State University

SJSU ScholarWorks

Faculty Publications, Environmental Studies

Environmental Studies

8-11-2015

\title{
Host promiscuity in symbiont associations can influence exotic legume establishment and colonization of novel ranges
}

\author{
Metha M. Klock \\ Louisiana State University, metha.klock@sjsu.edu \\ Luke G. Barrett \\ CSIRO Agriculture Flagship \\ Peter $\mathrm{H}$. Thrall \\ CSIRO Agriculture Flagship \\ Kyle E. Harms \\ Louisiana State University
}

Follow this and additional works at: https://scholarworks.sjsu.edu/envs_pub

Part of the Terrestrial and Aquatic Ecology Commons

\section{Recommended Citation}

Metha M. Klock, Luke G. Barrett, Peter H. Thrall, and Kyle E. Harms. "Host promiscuity in symbiont associations can influence exotic legume establishment and colonization of novel ranges" Diversity and Distributions (2015): 1193-1203. https://doi.org/10.1111/ddi.12363

This Article is brought to you for free and open access by the Environmental Studies at SJSU ScholarWorks. It has been accepted for inclusion in Faculty Publications, Environmental Studies by an authorized administrator of SJSU ScholarWorks. For more information, please contact scholarworks@sjsu.edu. 


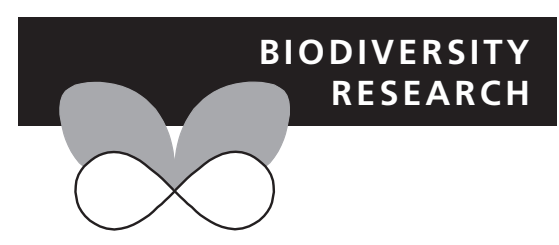

\title{
Host promiscuity in symbiont associations can influence exotic legume establishment and colonization of novel ranges
}

\author{
Metha M. Klock ${ }^{1 \star}$, Luke G. Barrett ${ }^{2}$, Peter H. Thrall ${ }^{2}$ and Kyle E. Harms ${ }^{1}$
}

${ }^{1}$ Department of Biological Sciences,

Louisiana State University, Baton Rouge, LA 70803, USA, ${ }^{2}$ CSIRO Agriculture Flagship, Canberra, ACT 2601, Australia

${ }^{*}$ Correspondence: Metha M. Klock, Department of Biological Sciences, Louisiana State University, Baton Rouge, LA 70803, USA.

E-mail: mklock1@tigers.lsu.edu

\begin{abstract}
Aim Invasive Acacia species have negatively impacted natural areas in multiple regions around the globe. Almost 400 Acacia species have been introduced outside their native ranges in Australia; approximately $6 \%$ have become invasive, $12 \%$ are naturalized, and $82 \%$ have no record of naturalization or invasion. This variation in invasiveness provides a comparative framework in which to examine mechanisms that either promote or constrain establishment and colonization of species in novel regions. Here, we experimentally examine the role that the legume-rhizobia symbiosis plays in the differential invasiveness of acacias introduced outside their native Australian ranges.
\end{abstract}

Location Canberra, Australia.

Methods We paired 12 Acacia species ranging in invasiveness globally with 12 rhizobial strains ranging in average symbiotic effectiveness. We measured plant growth and nodulation success and abundance to assess whether invasive acacias were more promiscuous hosts, that is had positive growth and nodulation responses to a broader range of rhizobial strains than naturalized and noninvasive species.

Results Invasive acacias had a higher growth response across more rhizobial strains (six of 12 strains) than naturalized and non-invasive species, but invasiveness categories differed only moderately with regard to the percentage of plants with nodules and nodulation abundance.

Main conclusion With respect to plant growth, invasive acacias appear to be more promiscuous hosts than naturalized and non-invasive Australian Acacia species. Plant growth response to nodulation, however, is likely more important than nodulation alone in the successful invasion of species in novel ranges. Results from this study help elucidate an important mechanism in the invasive capacity of legumes.

\section{Keywords}

Acacia, biological invasions, interactions, invasion, legume, mutualisms.

\section{INTRODUCTION}

Interactions between hosts and their mutualist partners, whether those partners are native or introduced, can play an important role in successful establishment and expansion outside of the hosts' native ranges. Plants are often dependent on specific mutualisms with insects and other animals for a diversity of functions, including pollination, seed dispersal, herbivore protection, etc. (Bascompte \& Jordano,
2007), and with fungi and bacteria (often symbionts) to acquire nutrients such as phosphorous and nitrogen, which might be otherwise unavailable (Richardson et al., 2000a). Positive species interactions take many forms and require empirical investigation to fully understand their roles in invasion of species introduced to novel ranges.

One feature likely to be critical to establishment of mutualistic interactions in novel environments is partner specificity. Several alternative hypotheses have been framed that 
make specific predictions in this regard. The Generalist Host Hypothesis suggests that invasive hosts are likely to be relative generalists in terms of associations with mutualists and are therefore less constrained than more specialized species by absence of specific partners (Parker, 2001; RodríguezEcheverría et al., 2011; Stanton-Geddes \& Anderson, 2011; Birnbaum et al., 2012). Species that are generalist hosts may be capable of associating with a wider range of symbionts in their novel range. The Enhanced Mutualism Hypothesis predicts that introduced species that become invasive form novel effective mutualisms with native species (Richardson et al., 2000a). Species that become invasive when introduced abroad may be more capable of forming novel mutualisms if they are less selective hosts. The Accompanying Mutualist Hypothesis posits that species that become invasive are introduced concurrent with mutualistic symbionts from their native ranges (Rodríguez-Echeverría, 2010). In all cases, the mutualistic association formed by the invader is presumed to contribute to a competitive advantage over native species.

An important mutualistic interaction that may play a role in invasion of certain species is between legumes (Family: Fabaceae) and nitrogen-fixing soil bacteria, or rhizobia. Legumes colonized by effective rhizobia gain access to nitrogen that would be otherwise unavailable to them; in turn, rhizobia receive photosynthate from their host plants (Sprent \& Sprent, 1990; Waters et al., 1998; Denison, 2000; Barrett et al., 2014). The legume-rhizobia mutualism plays a key role in determining plant productivity in native ecosystems (van der Heijden et al., 2008). In addition, legumes that find suitable symbionts may be more capable of establishment, growth and survival than those that do not (Thrall et al., 2000, 2005). This has been shown in both revegetation and agricultural settings where legumes inoculated with rhizobial strains known to be effective symbionts had higher rates of performance than those that were non-inoculated (Bullard et al., 2005; Thrall et al., 2005). For legumes introduced to novel regions, those finding rhizobial symbionts with which they can develop a mutualistic relationship may have an advantage over species that cannot.

Here we focus on invasive acacias as a model to test the role that specificity in mutualistic interactions might play in determining invasiveness. Acacia is a genus of legumes including a diverse range of species introduced around the globe that have become differentially invasive in novel ranges (Richardson et al., 2011). Acacias are native to Australia, where over 1000 species occur (Miller et al., 2011); species of Acacia have been introduced abroad for purposes such as forestry, agriculture, erosion control and ornamental display (Kull \& Rangan, 2008; Carruthers et al., 2011). Many of these species have subsequently escaped their intended use (Rejmánek \& Richardson, 2013) out-competing native species, increasing soil erosion, altering soil chemistry and facilitating invasion of non-native grasses (Richardson et al., 2000a; Richardson \& Kluge, 2008). An in-depth investigation of the global introduction status of Australian Acacia species by Richardson et al. (2011) (later updated by Rejmánek \&
Richardson [2013]) found that 384 Acacia species have been introduced to areas outside of Australia. Of these species, 22 ( $\sim 6 \%)$ have become invasive, $47(\sim 12 \%)$ naturalized with no record of invasiveness and $315(\sim 82 \%)$ with no record of naturalization or invasion. Global variation in invasiveness among Acacia species provides a large-scale opportunity to evaluate mechanisms that may limit or constrain their establishment when introduced to novel ranges.

Acacias and other legumes often differ in their ability to nodulate with different rhizobial strains (Thrall et al., 2000; Rodríguez-Echeverría et al., 2012). Thrall et al. (2000) found that less common acacias within Australia tended to be more specific rhizobial hosts than more widespread Acacia species. Moreover, population level variation in plant growth within certain Acacia species for the same rhizobial symbionts has also been observed (Burdon et al., 1999; Barrett et al., 2012). In a detailed study of two Acacia species (A. salicina and $A$. stenophylla) where each was paired with soils collected from its own and the other species' populations, Thrall et al. (2007) found considerable variation in host specificity between the two species, with $A$. salicina growing well regardless of soil origin, and A. stenophylla growing best in its own soils. Such variation in legume-host specificity with rhizobia may play an important role in determining invasiveness of different host species following introduction to novel ranges.

Introduced Acacia species that are more promiscuous hosts (capable of nodulating with a wider suite of rhizobial strains) may more readily find suitable symbionts as predicted by the Enhanced Mutualism Hypothesis (Richardson et al., 2000b), or be less selective with regard to rhizobial strains they form associations with in new environments, as predicted by the Generalist Host Hypothesis (RodríguezEcheverría, 2010; Birnbaum et al., 2012). Acacia species that become invasive may also be co-introduced to novel ranges with their native symbionts as predicted by the Accompanying Mutualist Hypothesis (Rodríguez-Echeverría et al., 2012; Crisóstomo et al., 2013; Ndlovu et al., 2013) and may suffer reduced performance if they do not encounter familiar symbionts (Wandrag et al., 2013). Promiscuous hosts may have a competitive advantage promoting establishment and colonization over native plant species; the latter could include both non-nitrogen fixing and more selective legume hosts. By investigating a suite of Acacia species that vary in invasiveness globally, we can assess whether host promiscuity has potential to differentially influence establishment and colonization of legumes in novel environments.

We hypothesized that rhizobial associations would influence invasiveness of Acacia species, with the following predictions: (1) invasive acacias will be more promiscuous hosts, developing effective symbiotic associations (based on plant growth and nodulation response) with a wider suite of rhizobial strains than naturalized or non-invasive Acacia species; and (2) both growth and nodulation (nodule presence/ absence, abundance) will be higher in invasive acacias. To test these predictions, we used the assignations of Richardson 
et al. (2011) to choose Acacia species from three categories of global invasiveness (invasive, naturalized and non-invasive) and then grew them with a genetically diverse set of rhizobial strains ranging in average symbiotic effectiveness from moderately to highly effective (as defined by Bever et al., 2013). We then compared symbiotic response (defined below) based on aboveground dry weight (biomass), as well as nodulation response to assess plant performance with different rhizobial strains. By comparing plant performance across a variety of strains ranging in effectiveness, we were able to examine host promiscuity of Acacia species varying in invasiveness abroad.

\section{METHODS}

\section{Acacia species}

We selected native Australian Acacia species from three categories of invasiveness, as defined by Richardson et al. (2011): (1) invasive outside Australia (22 species); (2) naturalized with no record of invasiveness (47 species); (3) recorded as introduced outside Australia with no record of naturalization (315 species). We chose four Acacia species from each category of invasiveness; particular Acacia species were selected based on evidence of widespread introduction and well-documented introduction histories. Invasive species chosen for this study are designated as some of the worst acacia invaders globally (Richardson \& Rejmánek, 2011). All species selected were those with records of repeated introductions abroad and included: (invasive) A. dealbata, A. longifolia, A. mearnsii, A. melanoxylon; (naturalized) A. cultriformis, A. murrayana, A. pendula, A. redolens; and (non-invasive) A. bivenosa, A. colei, A. hakeoides, A. stenophylla. These species vary in geographic distribution across Australia (Fig. 1). In addition to the criteria noted above, species were selected from each invasive category to represent both widespread $(>1000$ herbarium records: A. dealbata, A. longifolia, A. melanoxylon, A. murrayana, A. hakeoides, A. stenophylla; [AVH, 2014]) and limited distributions (< 1000 herbarium records: A. mearnsii, A. cultriformis, A. pendula, A. redolens, A. bivenosa, A. colei; $[\mathrm{AVH}, 2014]$ ) within their native range as there is at least some evidence that more widespread acacias may tend towards greater promiscuity (Thrall et al., 2000).

\section{Rhizobial strain preparation}

Bever et al. (2013) examined average effectiveness of 40 rhizobial strains with nine Acacia species native to Australia. Symbiotic effectiveness was based on plant growth response to inoculation with these different strains. They found extensive variation in symbiotic effectiveness of these strains; some strains were broadly effective across all Acacia species (as measured by aboveground biomass), some varied in effectiveness depending on Acacia species with which they were paired, while others were relatively ineffective symbionts with all Acacia species tested (i.e. low plant growth response among all Acacia species tested). We chose a subset of twelve strains from those evaluated by Bever et al. (2013) to represent variation in average effectiveness to challenge plants with diverse symbiotic conditions. Strains chosen represented three genera known to associate with Australian Acacia species: Bradyrhizobium, Rhizobium and Sinorhizobium (Lafay \& Burdon, 2001; Hoque et al., 2011) and were all originally isolated from Acacia species root nodules in Australia (Table S1 in Supporting Information).

To prepare inoculants, we streaked samples from cultures stored at $-80{ }^{\circ} \mathrm{C}$ individually on yeast mannitol agar plates. Plates were incubated at $28{ }^{\circ} \mathrm{C}$ for $7-12$ days until colony growth. Cultures were transferred to $50-\mathrm{ml}$ Falcon tubes containing yeast mannitol broth (YMB) and grown for 5-7 days until media became cloudy. Tubes were then centrifuged at approximately $5000 \times \mathrm{g}$ for $60 \mathrm{~min}$ until a pellet formed; YMB was decanted, and tubes were filled to $50 \mathrm{ml}$ with sterile water. We resuspended the pellet and measured optical density (OD) of the solution. Inoculants were adjusted to an OD of $2.0 \times 10^{7}$ cells $/ \mathrm{ml}$ through addition of sterile water. Identity of rhizobial strains selected from stored samples was confirmed via re-sequencing the $16 \mathrm{~S}$ rRNA gene prior to inoculating plant hosts.

\section{Glasshouse experiment}

We conducted a glasshouse inoculation experiment to examine symbiotic selectivity of Acacia species differing in invasiveness with rhizobia. Glasshouse and laboratory facilities were located at the CSIRO Agriculture Flagship site in Canberra, Australia. We obtained seeds from the Australian Seed Company and the Australian Tree Seed Centre in June 2013. All acacia seed was Australian in origin. Seeds were surface sterilized by soaking in $90 \%$ ethanol for $10 \mathrm{~s}, 10 \%(\mathrm{v} / \mathrm{v})$ sodium hypochloride for $5 \mathrm{~min}$ and washed in six changes of sterile water (Somasegaran \& Hoben, 1994). Seeds were scarified using a boiling water treatment (i.e. boiling water was poured over surface sterilized seeds), left to soak overnight at $4{ }^{\circ} \mathrm{C}$ and then sown in a mixture of autoclaved sand and soil (1:1 by volume). Germination media was autoclaved to ensure sterility. Seedlings were grown between 1 to 2 weeks in a laboratory growth chamber $\left(25^{\circ} \mathrm{C}\right)$ to avoid contamination by free-living bacteria. Seedlings were transferred from growth chamber to glasshouse facilities and transplanted in pots filled with steam-sterilized sand and vermiculite (1:1 by volume).

Seedlings were inoculated with individual rhizobial strains 3 days after transplanting. Ten replicates of each Acacia species were treated with $1 \mathrm{ml}(\mathrm{OD}=0.2)$ of inoculant of each strain. Two controls were also included; a nitrogen-free control in which non-inoculated plants were watered only with a nitrogen-free solution and sterile water (designated as $\mathrm{N}^{-}$) and a control in which non-inoculated plants were watered with a nutrient solution containing nitrogen (designated as $\mathrm{N}^{+}$). Nitrogen was provided to plants in the form of ammonium nitrate $\left(\mathrm{NH}_{4} \mathrm{NO}_{3}\right) \quad(6 \mathrm{~mm} /$ plant once a week). We 


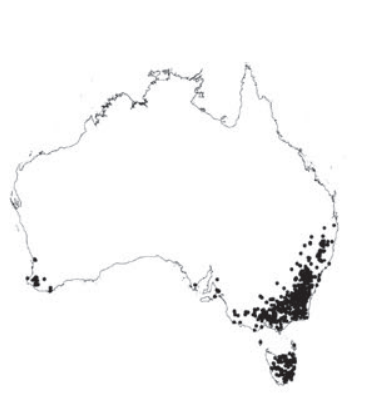

Acacia dealbata*

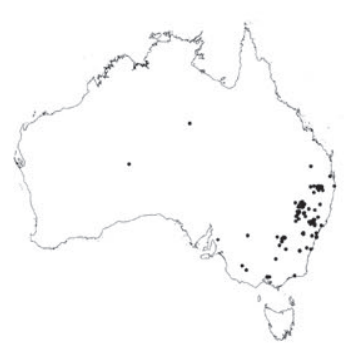

Acacia cultriformis

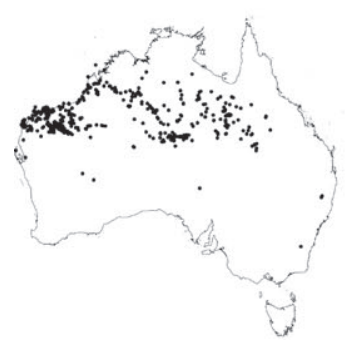

Acacia bivenosa

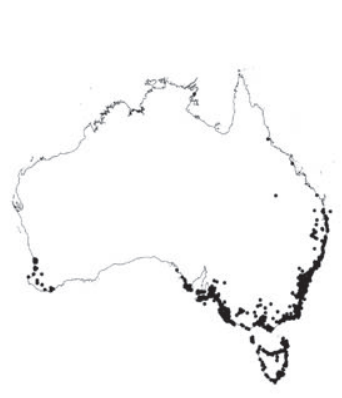

Acacia mearnsii

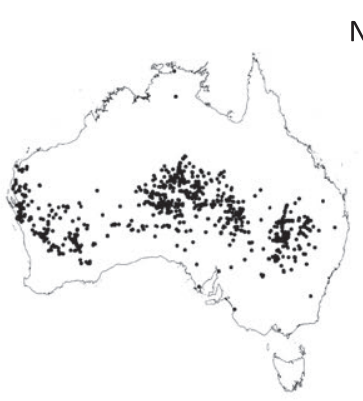

Acacia murrayana*

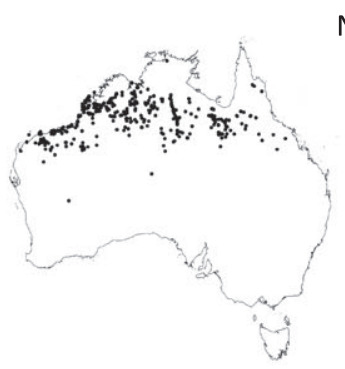

Acacia colei
Invasive

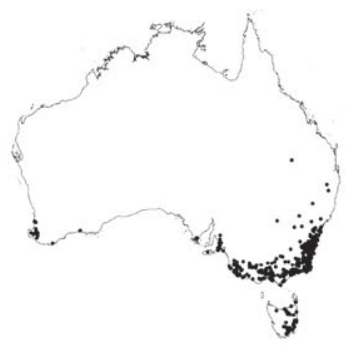

Acacia longifolia*

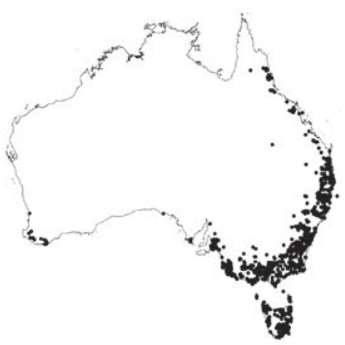

Acacia melanoxylon*

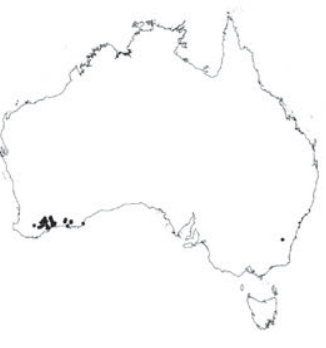

Acacia redolens

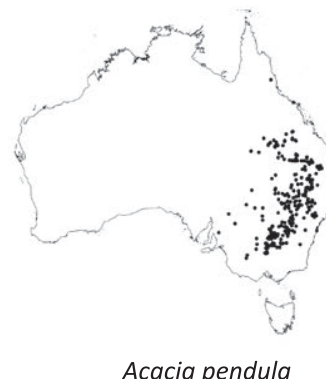

Non-invasive

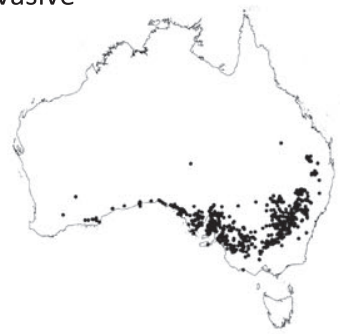

Acacia hakeoides*

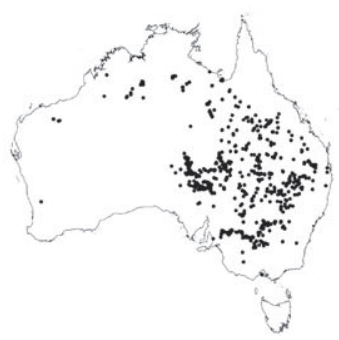

Acacia stenophylla*

Figure 1 Distribution maps of Acacia species used in this experiment within Australia (based on herbarium records from the Australian National Herbarium, Canberra, Australia [AVH, 2014]). '*' denotes widespread species (i.e. > 1000 herbarium records). All other species are limited in distribution (i.e. $<1000$ herbarium records).

separated rhizobial treatments by bench within the glasshouse to minimize potential for cross-contamination. Plants were grown for 18 weeks in a temperature-controlled glasshouse $\left(\sim 20^{\circ} \mathrm{C}\right)$ under natural light conditions. All plants except for the $\mathrm{N}^{+}$control were watered with sterile $\mathrm{N}$-free McKnights solution (1949) twice weekly and with sterile water as necessary. $\mathrm{N}^{+}$control plants were watered with McKnight's solution amended with ammonium nitrate on the same watering schedule as all other plants.

Plants were harvested in October 2013. During harvest, plants were clipped at the soil surface and aboveground material was placed in paper bags. Roots were scored for nodulation quantity $(0,<10,10-50,>50)$ and quality [none, ineffective (black or very small white nodules), intermediate (mixture of small to medium white/pink nodules), good (pink nodules) and very good (large nodules with pink/red centres)] (Thrall et al., 2011). Aboveground material was oven-dried at $70{ }^{\circ} \mathrm{C}$ for $48 \mathrm{~h}$ and weighed.

\section{Statistical analysis}

We measured response of acacias in three invasiveness categories and as individual hosts to inoculation with 12 rhizobial strains. Although not the explicit focus of this study, we also examined response of Acacia species that are either widespread or limited in native range distribution to 12 rhizobial strains. Differences among invasiveness categories and between range distributions to individual strains were measured by assessing host symbiotic response (based on aboveground biomass; defined below), presence/absence of nodules and nodulation index of effectiveness (based on nodule quantity score categories). There was a low level of contamination of $\mathrm{N}^{-}$controls $(\sim 14 \%)$, and any control plants with nodules were excluded from analyses. Symbiotic response was calculated following methods of Thrall et al. (2011). We divided biomass of each invasive group/native distribution/ acacia host by average biomass of the non-inoculated control 
for that group/distribution/host. A value of 1 indicates that biomass for a particular group/distribution/host $\mathrm{x}$ strain combination did not differ from the $\mathrm{N}^{-}$control, a value of $>1$ indicates that the group/distribution/host performed better than its $\mathrm{N}^{-}$control for a particular strain, and a value of $<1$ indicates that the group/distribution/host performed worse than its $\mathrm{N}^{-}$control. Using this measure of symbiotic response to analyse plant responses rather than aboveground biomass allowed us to minimize any bias due to potential differences in intrinsic growth rates among Acacia species.

We examined these variables for the entire data set using generalized linear mixed models (GLMM) with Acacia species as a random effect to control for species-specific variation that may influence growth or nodulation response. We used GLMM because it allowed us to include variability in growth and nodulation response of individual Acacia species in our model, while using invasiveness category as our main predictor. In our models, symbiotic response and nodulation index have Gaussian distributions and nodule presence/absence has a binomial distribution with a logit link function. We also examined symbiotic response and nodule presence/ absence for individual host species to examine species-specific response to rhizobial strains. We used ANOVA to analyse symbiotic response for individual hosts across rhizobial strains and logistic regression to analyse nodule presence/absence. A post hoc pairwise t-test with a Bonferroni adjustment was used to compare symbiotic response of different host species to each rhizobial strain. For invasiveness and native range distribution categories and individual host species, $\mathrm{N}^{-}$controls were used as a standard to compare symbiotic response across strains. All analyses were conducted using $\mathrm{R}$ statistical programming language version 3.0.2 ( $\mathrm{R}$ Core Team 2013).

We used the $\mathrm{R}$ statistical package 'lme4' (Bates et al., 2012) to examine whether symbiotic response/nodulation presence/nodule index was affected by an interaction between invasive group/native range distribution and rhizobial strains, with Acacia species as a random effect. We used likelihood ratio testing to compare full and reduced models and to determine variables for inclusion in the model. Models with the lowest Akaike information criterion (AIC) score were selected for further analysis unless the difference in AIC values was $<10$, in which case models were considered equally likely (Burnham \& Anderson, 2002; Bolker et al., 2009). We used the $\mathrm{R}$ statistical package 'multcomp' (Hothorn et al., 2008) to examine whether there were differences in symbiotic response among invasiveness categories/range distributions for individual strains.

\section{RESULTS}

\section{Symbiotic response across invasiveness categories and host species}

We found a significant interaction between invasiveness category and strain for symbiotic response, indicating that
Table 1 Average symbiotic response (i.e. average biomass of each invasive group per rhizobial strain divided by the average biomass of the non-inoculated control for that group) for each invasiveness category among rhizobial strains. The invasive category had a significantly greater symbiotic response (marked with $a^{(*)}$ ) than the naturalized and non-invasive categories for six strains. Naturalized and non-invasive categories did not differ significantly in symbiotic response among strains. Strains marked as B, Bradyrhizobium; R, Rhizobium and S, Sinorhizobium.

\begin{tabular}{lccl}
\hline Strain & Invasive & Naturalized & Non-invasive \\
\hline 01B & $36.83^{*}$ & 5.62 & 8.46 \\
02B & $34.38^{*}$ & 4.01 & 6.79 \\
03B & $61.40^{*}$ & 12.87 & 7.98 \\
04B & $48.41^{*}$ & 9.79 & 8.82 \\
05S & 1.76 & 1.64 & 1.50 \\
06B & 1.32 & 1.41 & 1.57 \\
07R & $37.70^{*}$ & 4.60 & 5.68 \\
08B & $5.96^{*}$ & 1.90 & 2.43 \\
09S & 1.49 & 1.16 & 3.47 \\
10S & 2.73 & 1.82 & 3.42 \\
11S & 2.73 & 1.14 & 2.88 \\
12R & 3.19 & 1.65 & 5.01 \\
\hline
\end{tabular}

growth response of a particular invasive group was dependent on the strain with which it was grown (Table S2). When compared across invasiveness categories, the invasive group had a significantly higher symbiotic response than naturalized or non-invasive groups for six of 12 strains (Tables 1 and S3). Naturalized and non-invasive groups did not differ significantly in symbiotic response across strains. When examined within invasiveness categories, the invasive group had a significantly higher symbiotic response than its $\mathrm{N}^{-}$control for five strains, followed by non-invasive (four strains) and naturalized groups (two strains) (Fig. 2).

We also found a significant interaction between host species and strain for symbiotic response (Table S4), indicating that (as shown in earlier studies) individual hosts have differential growth responses depending on rhizobial strains with which they are grown. Host species in different invasiveness categories varied in consistency of their responses to individual rhizobial strains. For example, hosts within the invasive group responded similarly, with a significantly higher symbiotic response to the same four to five rhizobial strains than naturalized and/or non-invasive species. Host species in the naturalized group varied in symbiotic response across strains, but consistently associated with fewer strains than invasive species. One species, A. murrayana, responded poorly to all strains with which it was paired. Host species in the non-invasive group varied the most in their symbiotic responses, with one species clearly responding positively to multiple strains, two species moderately and one species poorly to all strains with which it was inoculated (Fig. S1 and Table S5). 


\section{Symbiotic response across native range distributions}

We did not find a significant interaction between native habitat distribution and strain for symbiotic response (Table S6). Acacia species that are widespread in their native range ( $>1000$ herbarium records) and more limited in distribution $(<1000$ herbarium records) did not differ in symbiotic response for individual strains (Fig. 3). The best models based on AIC values indicated that symbiotic response of acacias with different range distributions varied based on strains with which they were grown as well as differences in range within their native continent.

\section{Nodulation response across invasiveness categories and host species}

We found a significant interaction between invasiveness category and strain for nodulation presence (Table S7) indicating that nodulation presence for an individual invasiveness category was dependent on the strain with which the invasive group was grown. While nodulation presence differed within individual invasiveness categories, it only differed for one strain among invasiveness categories. In other words, for each individual rhizobial strain tested except one, we found no significant difference in nodulation presence among invasiveness categories. When examined individually, invasive and naturalized groups showed $50 \%$ or greater nodulation for eight strains and non-invasive group for ten strains (Fig. 4).

Individual host species differed in nodulation presence depending on the strain with which they were paired. In general, nodulation success was high; all species showed $50 \%$ or greater nodulation for eight or more strains, except for two species: A. colei (only one strain $>50 \%$ ) and $A$. murrayana (three strains $>50 \%$ ) (Fig. S2). For species that had a high symbiotic response, nodulation was consistently high. However, our results also showed that effective nodulation does not always translate to a high symbiotic response.

We found a significant interaction between invasiveness category and strain for nodulation index (Table S8). The invasive group had a higher nodulation index than naturalized and non-invasive groups for the same two strains. For all other strains, nodulation index did not differ significantly among invasiveness categories (Fig. S3).

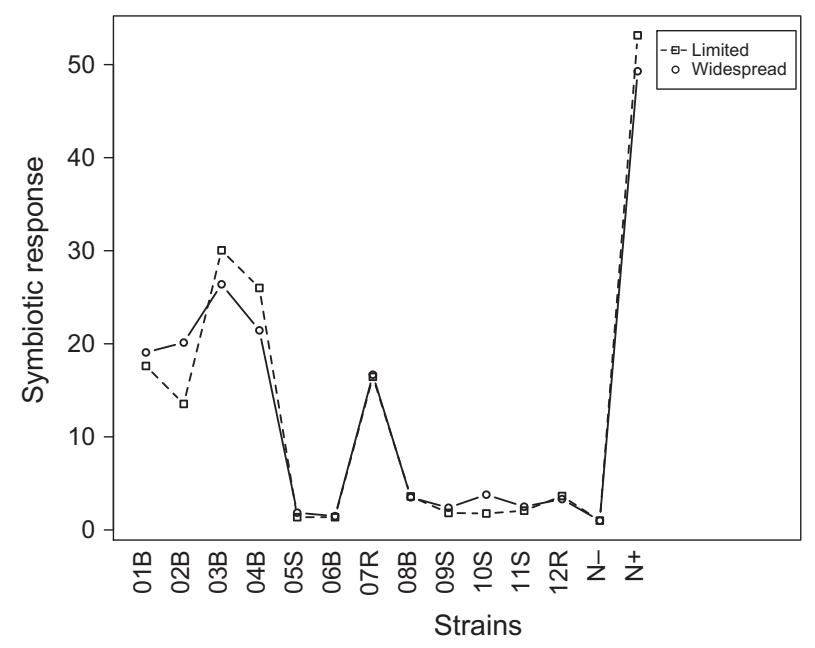

Figure 3 Symbiotic response for species in limited versus widespread native range distributions among rhizobial strains. Strains marked as B, Bradyrhizobium; R, Rhizobium and S, Sinorhizobium.
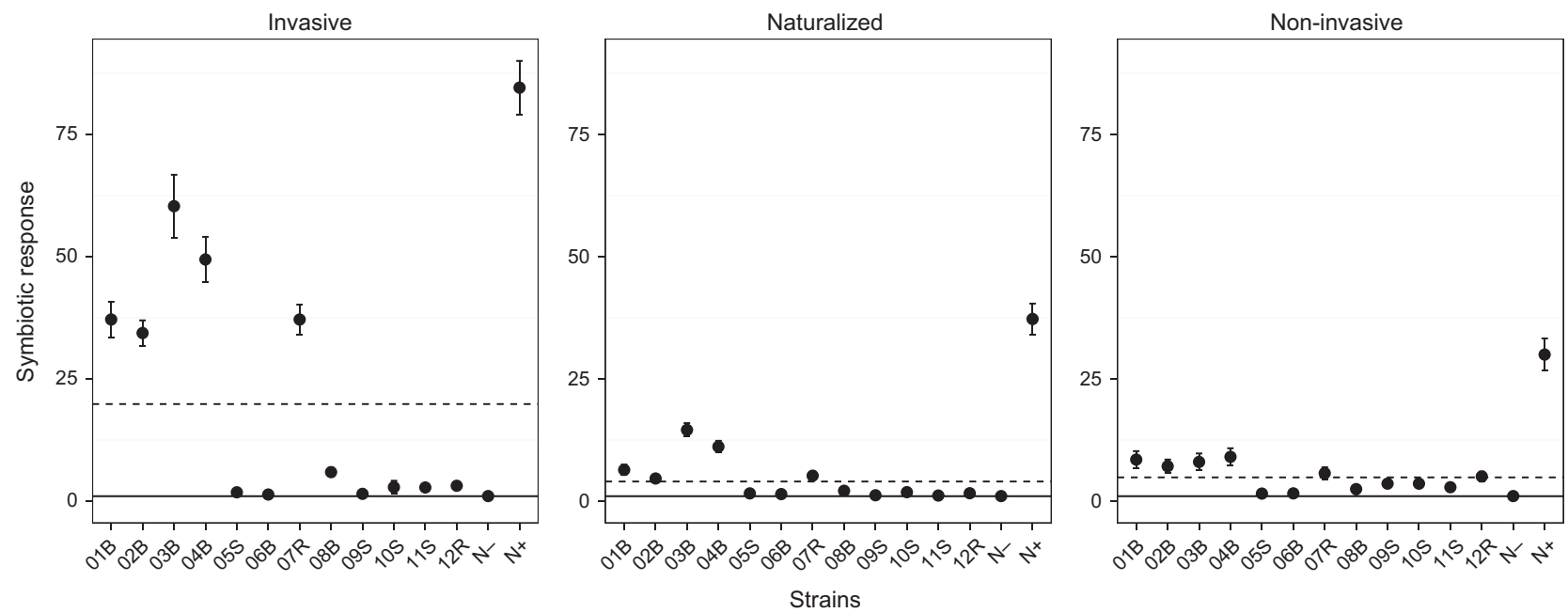

Figure 2 Symbiotic response of invasiveness categories to different rhizobial strains. The horizontal solid line at 1 indicates the point at which host species within a given invasiveness category has the same symbiotic response as the $\mathrm{N}^{-}$control. The dashed line is the average symbiotic response for all host species within a given invasiveness category combined across all strains. Points above the solid line indicate a positive symbiotic response, and points below the solid line indicate a negative symbiotic response. Error bars represent standard errors (SE) of the means. Strains marked as B, Bradyrhizobium; R, Rhizobium and S, Sinorhizobium. 

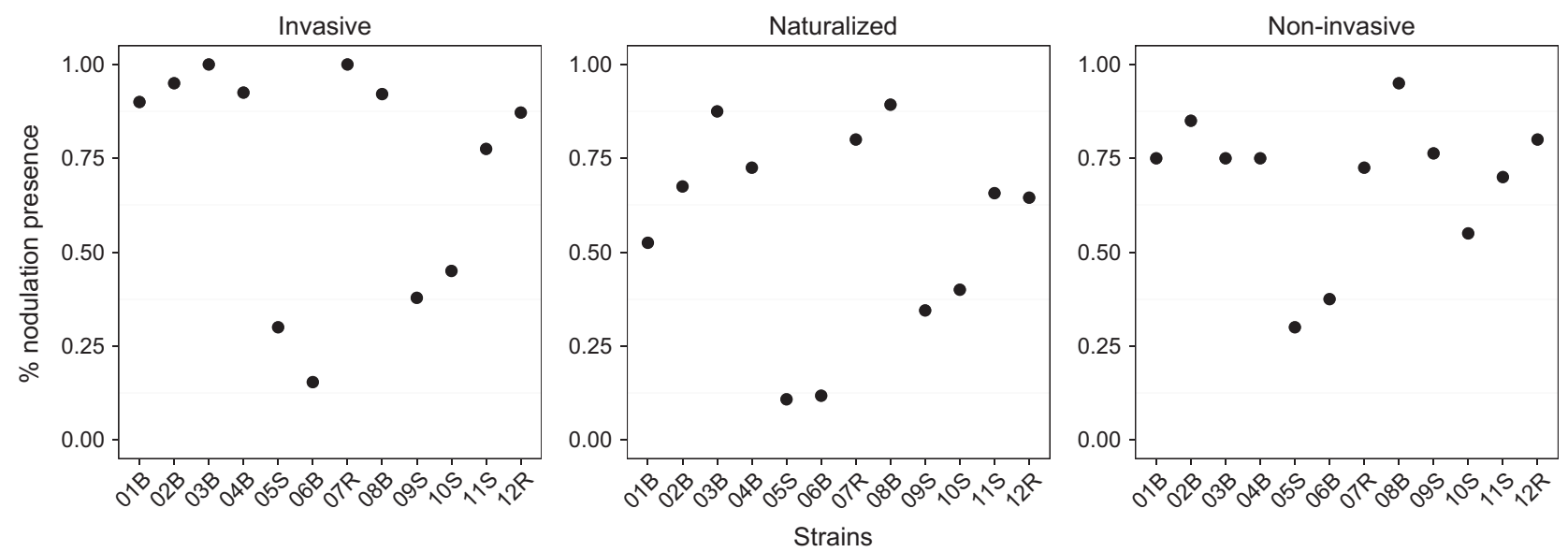

Figure 4 Per cent nodulation for each invasiveness category among rhizobial strains. Strains marked as B, Bradyrhizobium; R, Rhizobium and S, Sinorhizobium.

\section{Nodulation response across native range distributions}

We found a significant interaction between native habitat distribution and strain for nodulation presence (Table S9). Models predicting nodulation index that included native habitat distribution, strain and an interaction between the two received similar support (Table S10). Acacia species that vary in distribution did not differ in nodule presence for individual strains (Fig. 4) and differed in nodule index for only one strain.

\section{DISCUSSION}

The goal of this study was to examine whether invasive acacias are more promiscuous hosts with regard to their rhizobial symbionts than naturalized and non-invasive acacias. We found that Acacia species categorized as invasive were able to effectively associate with a significantly greater number of arbitrarily selected rhizobial strains than species that have so far proven to be non-invasive. This is important because acacias that are more promiscuous hosts may more readily find suitable symbionts when they are introduced to novel ranges, hence facilitating their establishment and subsequent range expansion (Rodríguez-Echeverría et al., 2009). Fidelity for specific rhizobial partners may thus be an important trait contributing to variation in species invasiveness.

Invasive Acacia species had more consistent patterns of positive symbiotic response to rhizobial strains with which they were paired (i.e. all invasive Acacia species had a significantly greater symbiotic response than their $\mathrm{N}^{-}$control for the same five strains), whereas species within naturalized and non-invasive groups were more variable in their response to different strains. This indicates that Acacia species that become invasive are more generally able to associate with a broader set of rhizobial strains, supporting our hypothesis that invasive acacias are generally more promiscuous hosts.
Our results are consistent with findings of Birnbaum et al. (2012), who examined the ability of multiple invasive Acacia species to develop effective symbiotic associations with rhizobia from soils in which hosts do not naturally occur in Australia. Invasive Acacia species were not limited in plant growth response when grown with biota from non-native soils, although rhizobial community composition did vary among sites sampled. In contrast, Wandrag et al. (2013) examined the acacia-rhizobia symbiosis using three host species introduced into New Zealand that vary in invasiveness and found that Acacia species growing in unfamiliar soils in their introduced range suffered reduced growth and nodulation, regardless of invasive status. Acacias in Australia may be more likely to find compatible rhizobial symbionts (i.e. those that are more closely related to their native symbiotic strains) on their native continent, even in unfamiliar soils, than in more geographically distant regions such as New Zealand, which may be driving the difference in results seen between these two studies. Although not the specific focus of this study, our results differed from those of Thrall et al. (2000), who found that less common acacias within Australia tended to be more specific rhizobial hosts than more widespread Acacia species. Further research is needed to examine the extent to which geographic origin of rhizobial strains influences Acacia species symbiotic responses.

Other mutualistic factors besides promiscuity may play a role in influencing invasion success of Acacia species abroad. In particular, recent studies have indicated that some invasive Acacia species have been introduced to novel ranges concurrent with their native rhizobial symbionts (RodríguezEcheverría, 2010; Crisóstomo et al., 2013; Ndlovu et al., 2013). Widespread distribution of other mutualistic soil organisms may also influence invasive success of introduced plant species. Schwartz et al. (2006) highlighted the potential unintended consequences of introduction of mycorrhizal fungi outside their native range as inoculum for agricultural and restoration purposes. Wide-scale introduction of symbiotic rhizobial and mycorrhizal organisms may increase 
probability of non-native species encountering their native symbionts in their novel range (Schwartz et al., 2006). Cointroduction of symbiotic organisms bypasses the need for species to develop novel associations with new partners and may facilitate rapid establishment and colonization of these species. Additional research is necessary to examine whether co-introduction of symbionts is a widespread phenomenon in acacia introduction history. However, we note that even in the case of co-introductions, ability to effectively associate with a broad range of rhizobia may be important, as studies have shown that within-species variability in response to different rhizobial strains can limit growth of some Acacia species (Burdon et al., 1999).

Even though our results support the idea that more invasive species are more promiscuous hosts, it is possible that reduction in suitable symbionts due, for example, to greater distance in relatedness between rhizobia from more widely distributed regions, will limit growth of even the more promiscuous Acacia species. We note that all rhizobial strains used in this study were Australian in origin. Future work would benefit from examining growth responses of additional Acacia species to native rhizobial strains, as well as additional strains from regions where these species are invasive. Acacia species may be more likely to associate and have a beneficial growth response with rhizobial strains that occur in their native range, even if they do not normally occur with those strains. Recent evidence has shown that Acacia species that are invasive outside of their native ranges within Australia are still able to find suitable rhizobial symbionts in regions where they were introduced (Birnbaum et al., 2012); however, effectiveness of this symbiosis may vary when acacias associate with more distantly related rhizobial strains (RodríguezEcheverría et al., 2012). Bacterial strains that occur in the same region may be more closely related to one another; hence, plant hosts may be more capable of developing effective symbioses with strains occurring within their native range (but see Barrett et al., 2012). In a recent examination of fungal communities occurring with Acacia species that have become invasive within their native continent of Australia, Birnbaum et al. (2014) found that, of the four Acacia species examined, three species associated with similar soil fungal communities, including symbiotic mycorrhizal fungi, in their native and invaded populations. This suggests that soil fungal, and possibly also soil bacterial communities within these hosts' native continent can be broadly distributed, and plant species may encounter the same or closely related strains in unfamiliar habitats within their larger continental range (Rodríguez-Echeverría et al., 2012). Examining growth responses of Acacia species when paired with rhizobial strains with which they occur in their introduced range may allow us to determine whether patterns of symbiotic response found here hold true on a larger geographic scale and provide valuable insight towards potential differences in symbiotic effectiveness with more distantly related rhizobial strains.

As in all short-term ecological experiment conducted in controlled environments, it is useful to consider the extent to which their results are relevant to long-term, real-world conditions. Our experiment examined plant growth response over a three-month period, covering early phase growth of different Acacia species. It has been shown that seedling and juvenile phases are key for establishment and survival of plants in challenging environments (Thrall et al., 2005; Barrett et al., 2012). Therefore, measuring plant performance over this early growth phase is likely to accurately predict relative establishment success of Acacia species in areas where they are introduced abroad.

Level of nodulation did not differ greatly among invasiveness categories, nor did nodulation index, and was generally high for all groups. For specific hosts, however, nodulation success (as measured by presence of nodules) differed, with certain species nodulating successfully with a greater number of strains than others. These results combined with symbiotic response results indicate that, whereas species in all invasiveness categories are capable of developing symbiotic associations with different rhizobial strains, those associations may not translate into an effective symbiotic response. In other words, even though species in different invasiveness categories can develop nodules with multiple strains, nodulation presence per se does not necessarily translate to a higher growth response. Effective rhizobial association may require nodulation, but then additional factors may determine overall effectiveness of the association, that is the extent to which the association confers increased plant performance and thus potential for population expansion.

Our study supports the hypothesis that host promiscuity with rhizobia, with regard to symbiotic response, is one mechanism that contributes to Acacia species invasion. Invasive Acacia species were more consistently able to form effective symbiotic relationships with more rhizobial strains than naturalized and non-invasive species, suggesting that they are less constrained in finding suitable symbiotic rhizobial partners when introduced abroad. However, some non-invasive Acacia species (e.g. A. bivenosa and A. stenophylla) were also promiscuous hosts. It may be that fewer individuals of these species have been introduced abroad (i.e. lower propagule pressure) and that limited propagules constrained their colonization and expansion in novel regions (Williamson, 1996; Simberloff, 2009). These species, which are currently less widespread on a global scale, should be monitored closely for further expansion.

When Acacia species are introduced outside their native range and subsequently escape intended use they have significant potential for negative impacts in both natural and managed environments (Yelenik et al., 2004; Gaertner et al., 2009). A relatively small proportion of Acacia species introduced outside their native range are currently recognized as invasive (Richardson et al., 2011); however, many more species have become naturalized and may have the potential to become invasive. Our results highlight the importance of monitoring and stopping intentional movement of naturalized and non-invasive Acacia species, particularly that are promiscuous hosts, to avoid potential future invasion of 
these species. Acacia species continue to be introduced around the world for purposes such as ornamental trade, timber, wood-fuel, etc. (Kull et al., 2011). Our research underscores the need to test species before introducing them to novel regions to determine whether they are promiscuous hosts, thereby circumventing further spread. In their native range, however, many Acacia species are commonly used to restore degraded lands (Murray et al., 2001), and promiscuous hosts may be excellent candidates for use in restoration projects.

\section{ACKNOWLEDGEMENTS}

We thank Mohammad S. Hoque, Kristy Lam, Holly Vuong, Alexandre de Menenzes, Kelly Hamonts and Elizabeth Weltman for help and guidance in the laboratory and glasshouse. We thank Meredith Blackwell, James T. Cronin, Bret Elderd and Richard Stevens for support and insight throughout development of this project. We thank Sandra P. Galeano and Katherine Hovanes for discussion in manuscript preparation. We thank three anonymous referees for helpful comments that improved the manuscript. The work was supported by a Louisiana Board of Regents EPSCoR grant and funding from the National Science Foundation (DEB1311290).

\section{REFERENCES}

AVH (2014) Australia's Virtual Herbarium, Council of Heads of Australasian Herbaria. Available at: http://avh.chah.org.au (accessed 3 November 2014).

Barrett, L.G., Broadhurst, L.M. \& Thrall, P.H. (2012) Geographic adaptation in plant-soil mutualisms: tests using Acacia spp. and rhizobial bacteria. Functional Ecology, 26, 457-468.

Barrett, L.G., Bever, J.D., Bissett, A. \& Thrall, P.H. (2014) Partner diversity and identity impacts on plant productivity in Acacia-rhizobial interactions. Journal of Ecology, 103, 130-142.

Bascompte, J. \& Jordano, P. (2007) Plant-animal mutualistic networks: the architecture of biodiversity. Annual Review of Ecology Evolution and Systematics, 38, 567-593.

Bates, D., Maechler, M. \& Bolker, B. (2012) Ime4: Linear mixed-effects models using $S 4$ classes. R package version 0.999999-0. Available at: http://CRAN.R-project.org/package $=$ lme4 (accessed 5 March 2014).

Bever, J.D., Broadhurst, L.M. \& Thrall, P.H. (2013) Microbial phylotype composition and diversity predicts plant productivity and plant-soil feedbacks. Ecology Letters, 16, 167-174. Birnbaum, C., Barrett, L.G., Thrall, P.H. \& Leishman, M.R. (2012) Mutualisms are not constraining cross-continental invasion success of Acacia species within Australia. Diversity and Distributions, 18, 962-976.

Birnbaum, C., Bissett, A., Thrall, P.H. \& Leishman, M.R. (2014) Invasive legumes encounter similar soil fungal communities in their non-native and native ranges in Australia. Soil Biology and Biochemistry, 76, 210-217.
Bolker, B.M., Brooks, M.E., Clark, C.J., Geange, S.W., Poulsen, J.R., Stevens, M.H.H. \& White, J.-S.S. (2009) Generalized linear mixed models: a practical guide for ecology and evolution. Trends in Ecology and Evolution, 24, 127-135.

Bullard, G.K., Roughley, R.J. \& Pulsford, D.J. (2005) The legume inoculant industry and inoculant quality control in Australia: 1953-2003. Australian Journal of Experimental Agriculture, 45, 127-140.

Burdon, J.J., Gibson, A.H., Searle, S.D., Woods, M.J. \& Brockwell, J. (1999) Variation in the effectiveness of symbiotic associations between native rhizobia and temperate Australian Acacia: within-species interactions. Journal of Applied Ecology, 36, 398-408.

Burnham, K.P. \& Anderson, D.R. (2002) Model selection and multimodal inference: a practical information-theoretic approach. Springer-Verlag, New York.

Carruthers, J., Robin, L., Hattingh, J.P., Kull, C.A., Rangan, H. \& van Wilgen, B.W. (2011) A native at home and abroad: the history, politics, ethics and aesthetics of acacias. Diversity and Distributions, 17, 810-821.

Crisóstomo, J.A., Rodríguez-Echeverría, S. \& Freitas, H. (2013) Co-introduction of exotic rhizobia to the rhizosphere of the invasive legume Acacia saligna, an intercontinental study. Applied Soil Ecology, 64, 118-126.

Denison, R.F. (2000) Legume sanctions and the evolution of symbiotic cooperation by rhizobia. The American Naturalist, 156, 567-576.

Gaertner, M., Den Breeÿen,, A., Hui, C. \& Richardson, D.M. (2009) Impacts of alien plant invasions on species richness in Mediterranean-type ecosystems: a meta-analysis. Progress in Physical Geography, 33, 319-338.

van der Heijden, M.G.A., Bardgett, R.D. \& Van Straalen, N.M. (2008) The unseen majority: soil microbes as drivers of plant diversity and productivity in terrestrial ecosystems. Ecology Letters, 11, 296-310.

Hoque, M.S., Broadhurst, L.M. \& Thrall, P.H. (2011) Genetic characterization of root-nodule bacteria associated with Acacia salicina and A. stenophylla (Mimosaceae) across south-eastern Australia. International Journal of Systematic and Evolutionary Microbiology, 61, 299-309.

Hothorn, T., Bretz, F. \& Westfall, P. (2008) Simultaneous inference in general parametric models. Biometric Journal, 50, 346-363.

Kull, C.A. \& Rangan, H. (2008) Acacia exchanges: wattles, thorn trees, and the study of plant movements. Geoforum, 39, 1258-1272.

Kull, C.A., Shackleton, C.M., Cunningham, P.J., Ducatillon, C., Dufour-Dror, J.-M., Esler, K.J., Friday, J.B., Gouveia, A.C., Griffin, A.R., Marchante, E., Midgley, S.J., Pauchard, A., Rangan, H., Richardson, D.M., Rinaudo, T., Tassin, J., Urgenson, L.S., von Maltitz, G.P., Zenni, R.D. \& Zylstra, M.J. (2011) Adoption, use and perception of Australian acacias around the world. Diversity and Distributions, 17, 822-836.

Lafay, B. \& Burdon, J.J. (2001) Small-subunit rRNA genotyping of rhizobia nodulating Australian Acacia spp. Applied and Environmental Microbiology, 67, 396-402. 
Miller, J.T., Murphy, D.J., Brown, G.K., Richardson, D.M. \& González-Orozco, C.E. (2011) The evolution and phylogenetic placement of invasive Australian Acacia species. Diversity and Distributions, 17, 848-860.

Murray, B.R., Thrall, P.H. \& Woods, M.J. (2001) Acacia species and rhizobial interactions: implications for restoration of native vegetation. Ecological Management \& Restoration, 2, 213-219.

Ndlovu, J., Richardson, D.M., Wilson, J.R.U. \& Le Roux, J.J. (2013) Co-invasion of South African ecosystems by an Australian legume and its rhizobial symbionts. Journal of Biogeography, 40, 1240-1251.

Parker, M.A. (2001) Mutualism as a constraint on invasion success for legumes and rhizobia. Diversity and Distributions, 3, 125-136.

R Core Team (2013) R: A language and environment for statistical computing. R Foundation for Statistical Computing, Vienna, Austria. Available at: http://www.R-project.org/ (accessed 2 December 2013).

Rejmánek, M. \& Richardson, D.M. (2013) Trees and shrubs as invasive alien species - 2013 update of the global database. Diversity and Distributions, 19, 1093-1094.

Richardson, D.M. \& Kluge, R.L. (2008) Seed banks of invasive Australian Acacia species in South Africa: role in invasiveness and options for management. Perspectives in Plant Ecology, Evolution and Systematics, 10, 161-177.

Richardson, D.M. \& Rejmánek, M. (2011) Trees and shrubs as invasive alien species - a global review. Diversity and Distributions, 17, 788-809.

Richardson, D.M., Allsopp, N., D’Antonio, C.M., Milton, S.J. \& Rejmánek, M. (2000a) Plant invasions - the role of mutualisms. Biological Reviews, 75, 65-93.

Richardson, D.M., Pyšek, P., Rejmánek, M., Barbour, M.G., Panetta, F.D. \& West, C.J. (2000b) Naturalization and invasion of alien plants: concepts and definitions. Diversity and Distributions, 6, 93-107.

Richardson, D.M., Carruthers, J., Hui, C., Impson, F.A.C., Miller, J.T., Robertson, M.P., Rouget, M., Le Roux, J.J. \& Wilson, J.R.U. (2011) Human-mediated introductions of Australian acacias - a global experiment in biogeography. Diversity and Distributions, 17, 771-787.

Rodríguez-Echeverría, S. (2010) Rhizobial hitchhikers from Down Under: invasional meltdown in a plant-bacteria mutualism? Journal of Biogeography, 37, 1611-1622.

Rodríguez-Echeverría, S., Crisóstomo, J., Nabais, C. \& Freitas, H. (2009) Belowground mutualists and the invasive ability of Acacia longifolia in coastal dunes of Portugal. Biological Invasions, 11, 651-661.

Rodríguez-Echeverría, S., Le Roux, J.J., Crisóstomo, J. \& Ndlovu, J. (2011) Jack-of-all trades and master of many? How does associated rhizobial diversity influence the colonization success of Australian Acacia species? Diversity and Distributions, 17, 946-957.

Rodríguez-Echeverría, S., Fajardo, S., Ruiz-Díez, B. \& Fernández-Pascual, M. (2012) Differential effectiveness of novel and old legume-rhizobia mutualisms: implications for invasion by exotic legumes. Oecologia, 170, 253261.

Schwartz, M.W., Hoeksema, J.D., Gehring, C.A., Johnson, N.C., Klironomos, J.N., Abbott, L.K. \& Pringle, A. (2006) The promise and the potential consequences of the global transport of mycorrhizal fungal inoculum. Ecology Letters, 9, 501-515.

Simberloff, D. (2009) The role of propagule pressure in biological invasions. Annual Review of Ecology Evolution and Systematics, 40, 81-102.

Somasegaran, P. \& Hoben, H.J. (1994) Handbook for rhizobia: methods in legume-rhizobium technology. Springer-Verlag, New York.

Sprent, J.I. \& Sprent, P. (1990) Nitrogen fixing organisms: pure and applied aspects. Cambridge University Press, Cambridge.

Stanton-Geddes, J. \& Anderson, C.G. (2011) Does a facultative mutualism limit species range expansion? Oecologia, 167, 149-155.

Thrall, P.H., Burdon, J. \& Woods, M.J. (2000) Variation in the effectiveness of symbiotic associations between native rhizobia and temperate Australian legumes: interactions within and between genera. Journal of Applied Ecology, 37, 52-65.

Thrall, P.H., Millsom, D.A., Jeavons, A.C., Waayers, M., Harvey, G.R., Bagnall, D.J. \& Brockwell, J. (2005) Seed inoculation with effective root-nodule bacteria enhances revegetation success. Journal of Applied Ecology, 42, 740-751.

Thrall, P.H., Slattery, J.F., Broadhurst, L.M. \& Bickford, S. (2007) Geographic patterns of symbiont abundance and adaptation in native Australian Acacia-rhizobia interactions. Journal of Ecology, 95, 1110-1122.

Thrall, P.H., Laine, A.-L., Broadhurst, L.M., Bagnall, D.J. \& Brockwell, J. (2011) Symbiotic effectiveness of rhizobial mutualists varies in interactions with native australian legume genera. PLoS One, 6, 1-11.

Wandrag, E.M., Sheppard, A., Duncan, R.P. \& Hulme, P.E. (2013) Reduced availability of rhizobia limits the performance but not invasiveness of introduced Acacia. Journal of Ecology, 101, 1103-1113.

Waters, J.K., Hughes, B.L. II, Purcell, L.C., Gerhardt, K.O., Mawhinney, T.P. \& Emerich, D.W. (1998) Alanine, not ammonia, is excreted from N2-fixing soybean nodule bacteroids. Proceedings of the National Academy of Sciences USA, 95, 12038-12042.

Williamson, M. (1996) Biological invasions. Chapman \& Hall, London.

Yelenik, S.G., Stock, W.D. \& Richardson, D.M. (2004) Ecosystem level impacts of invasive Acacia saligna in the South African fynbos. Restoration Ecology, 12, 44-51.

\section{SUPPORTING INFORMATION}

Additional Supporting Information may be found in the online version of this article: 
Figure S1 Symbiotic response of individual host species to different rhizobial strains.

Figure S2 Per cent nodulation for each host species x rhizobial strain combination.

Figure S3 Average nodulation index for different invasiveness categories among rhizobial strains.

Table S1 Original Acacia species hosts for each rhizobial strain used in growth experiments.

Table S2 GLMM models predicting difference in symbiotic response among invasiveness categories.

Table S3 Average biomass (g) of each invasiveness category $\mathrm{x}$ rhizobial strain.

Table S4 ANOVA results for effects of host species x rhizobial strain on symbiotic response.

Table S5 Average aboveground biomass (g) of each Acacia species x rhizobial strain.

Table S6 GLMM models predicting difference in symbiotic response among native range distributions.

Table S7 GLMM models predicting difference in nodulation presence among invasiveness categories.
Table S8 GLMM models predicting difference in nodulation index among invasiveness categories.

Table S9 GLMM models predicting difference in nodulation presence among Australian range distributions.

Table S10 GLMM models predicting difference in nodulation index among Australian range distributions.

\section{BIOSKETCH}

Metha M. Klock is a doctoral candidate at Louisiana State University in Baton Rouge, LA. Her interests include understanding the mechanisms driving species invasions, best practices towards restoring disturbed native ecosystems and integrating people from diverse backgrounds in conserving native habitat.

Author contributions: M.M.K., L.G.B, P.H.T. and K.E.H. conceived of and developed the idea; M.M.K. and L.G.B. analysed the data; and M.M.K. led the writing with input from all authors.

Editor: Mark van Kleunen 